\title{
Inclusión y competición: Adaptaciones para disminuir las barreras de participación de un grupo de gimnastas con discapacidad intelectual en gimnasia rítmica y propuesta de reglamento específico para la competición \\ Inclusion and competition: adaptations to reduce barriers of participation of a group of gymnasts with intellectual disabilities in rhythmic gymnastics, and proposal of specific regulations for competitions
}

María José Montilla Reina

Universidad de Barcelona (España)

\begin{abstract}
Resumen. Se presenta una experiencia innovadora llevada a cabo en un club de gimnasia rítmica, con cuatro gimnastas con discapacidad intelectual, tres chicas y un chico de 16 a 21 años, con necesidades de apoyo intermitente. El grupo ha realizado dos sesiones semanales de 2 horas de entrenamiento de manera inclusiva, durante dos temporadas, con 15 gimnastas sin discapacidad, la intervención de una entrenadora y una especialista de apoyo. Los objetivos son exponer los ajustes que se han llevado a cabo para disminuir las barreras de participación en los entrenamientos inclusivos; participar en exhibiciones que realiza el club; crear y proponer a la Federación Catalana de Gimnasia un reglamento específico para gimnastas con discapacidad intelectual e incluirlos en las competiciones oficiales; y participar en competiciones organizadas por esta Federación. Se han llevado a cabo ajustes en el reglamento, en las estrategias didácticas, y en la organización de los entrenamientos, que han favorecido la participación, el aprendizaje y la ejecución de los ejercicios. Los resultados más relevantes son la participación en una exhibición formando parte de un grupo de gimnastas sin discapacidad; la participación en dos exhibiciones con un ejercicio específico con aros durante dos competiciones organizadas por la Federación Catalana de Gimnasia y la participación en la I Copa Catalana de gimnasia rítmica, en el nivel XI, con un ejercicio de aros y pelotas, junto con otros tres conjuntos de gimnastas con discapacidad intelectual. Remarcamos la satisfacción por parte de todas y todos los participantes en la experiencia. Palabras clave: Gimnasia rítmica, discapacidad intelectual, inclusión, adaptaciones, apoyos, competición.
\end{abstract}

\begin{abstract}
An innovative experience carried out in a rhythmic gymnastics club is presented, involving four gymnasts with intellectual disabilities - three girls and a boy between 16 and 21 years of age - who have intermittent support needs. The group participated in two weekly sessions of 2 hours of training in an inclusive manner, together with 15 gymnasts with no disabilities, and in presence of a coach and a support specialist. The aim was to present adaptations that had been made to reduce barriers to participating in inclusive trainings and in exhibitions held by the club; to create and propose to the Catalan Gymnastics Federation specific rules for gymnasts with intellectual disabilities that include a new level in official competitions; and to participate in competitions organised by the Federation. Adaptations and support were carried out in the organisation of the trainings, in the rules, and in the teaching strategies, which favoured participation, learning, and exercise execution. The most relevant results were the participation in an exhibition with gymnasts with no disabilities; participation in two exhibitions with specific exercises using hoops; participation in two competitions organised by the Catalan Gymnastics Federation; and participation in the $1^{\text {st }}$ Catalan Cup of rhythmic gymnastics, level XI, with an exercise with hoops and balls, in which they competed with three other groups of gymnasts with intellectual disabilities and 60 other clubs. We bring to attention the high level of satisfaction of all who participated in the experience.
\end{abstract}

Keywords: Rhythmic gymnastics, intellectual disability, inclusion, adaptations, support, competition.

\section{Introducción y justificación}

La práctica de actividad físico-deportiva (AFD) en personas con discapacidad es un derecho recogido en el Artículo 30 de la Convención Internacional de la ONU sobre los Derechos de las Personas con Discapacidad de 2006. Con el fin de participar en igualdad de condiciones en actividades recreativas, de esparcimiento y deportivas, los Estados han de tomar las medidas pertinentes para alentar y promover la participación en las actividades deportivas generales a todos los niveles y así asegurar que los niños y las niñas con discapacidad tengan igual acceso (ONU, 2006).

Existe una relación directa entre práctica de AFD y la mejora de la calidad de vida, como la autodeterminación, el bienestar físico y emocional, las relaciones interpersonales o la inclusión social (Shalock \& Verdugo, 2003). Asimismo, son conocidos los beneficios que las AFD producen en el desarrollo humano. Nadie discute que estar físicamente acti-

Fecha recepción: 18-12-18. Fecha de aceptación: 03-06-19 María Jose Montilla Reina mmontillar@gencat.cat vo es importante para el desarrollo de las personas, y aceptamos que la participación de niños y niñas con discapacidad en actividades físicas promueven y mejoran las aptitudes físicas y bienestar psicológico (Murphy \& Carbone, 2008). Pero la realidad es que tienen menos oportunidades de participación, lo que provoca un impacto negativo en su desarrollo personal.

Si analizamos los niveles de actividad física en el caso de los y las adolescentes sin discapacidad, estos se encuentran todavía lejos de las recomendaciones que realiza la Organización Mundial de la Salud (OMS), sobre todo las chicas de 13 años en adelante (Ramos, Jiménez-Iglesias, Rivera \& Moreno, 2016). Esta situación se agrava entre la población con discapacidad, y en concreto en los jóvenes con discapacidad intelectual. Los jóvenes con Síndrome de Down, en general, llevan una vida sedentaria y no realizan los 60 minutos recomendados por la OMS, según un estudio de Espósito, MacDonald, Hornyak \& Hulrich (2012). La misma conclusión se extrae del estudio realizado por Rodriìguez, Castillo, Redin \& Yanci, J. (2016), con 10 adolescentes con discapacidad intelectual en el País Vasco, que muestra que los niveles de AFD están muy por debajo de las recomenda- 
ciones de la OMS. El estudio también expone que el 70\% no realiza AFD entre las 18 y las 22 horas. Además, 2 de cada 3 adolescentes con discapacidad intelectual no realiza ningún tipo de AFD durante el fin de semana.

Por otra parte, nos interesa el enfoque inclusivo de las AFD y del deporte. El término inclusivo se está extendiendo e implica que todas y todos los participantes, con independencia de sus características y limitaciones, asistan y desarrollen las actividades en los mismos espacios que sus compañeros y compañeras, con los apoyos y adaptaciones individuales que sean necesarios (Van Copennolle, 2006).

Aunque la inclusión de niños y niñas con discapacidad en la educación es una tendencia general en Europa y España, la aparición y normalización de su imagen es poco presente todavía. Moya-Mata, Ruiz, Martin, Pérez \& Ros (2017), concluyen que apenas aparecen en los libros de texto de Educación Física en Primaria del sistema educativo español, siendo casi invisible la mujer con discapacidad. Aparecen sobre todo imágenes de chicas con discapacidad física, usuarias de sillas de ruedas, a las que se relacionan con el ámbito competitivo de máximo nivel, imagen muy alejada de la inclusión de la discapacidad en la sociedad actual y concretamente, de la discapacidad intelectual. Precisamente en el deporte y el ocio la exclusión todavía es algo habitual, cuando es extremadamente importante que se les dé las mismas oportunidades de participación en todos los aspectos de la vida, para alcanzar niveles similares en cuanto a su desarrollo integral, como así demuestran Pimenta, Zuchetto, Bastos \& Corredoira (2016) respecto a la evolución de 15 personas con Trastorno del Espectro Autista, en habilidades acuáticas tras un programa de natación.

Es indiscutible que estar físicamente activo es muy importante para el desarrollo y la mejora de la calidad de vida, pero no todo el mundo está convencido de los beneficios de incluir niños y niñas con discapacidad en estas actividades. Es necesario que todos los agentes que intervienen en el proceso de inclusión muestren comportamientos favorables hacia dicho proceso como es la participación activa y conjunta (Hutzler, 2003); Asimismo, Hutzler \& Korsensky (2010) consideran que las en las actividades se debe proporcionar un ambiente de colaboración, prestar atención a las familias y a la formación de las personas que las llevan a cabo. Lo que parece evidente es que un contacto más cercano, compartiendo deporte con personas con discapacidad puede mejorar las actitudes hacia la discapacidad, como demuestran Abellán, Sáez-Gallego \& Reina (2018) en un estudio con estudiantes de Educación Secundaria.

Aunque todavía encontramos barreras para poder participar en igualdad de condiciones respecto al resto de personas, destacando los costes elevados; la falta de conocimientos de los y las profesionales; la falta de programas específicos; las barreras emocionales y psicológicas; las barreras relacionadas con la información; la falta de confianza en los apoyos que necesitan; las barreras relacionadas con los equipamientos o el estigma social y actitudes negativas especialmente en medios de comunicación (Murphy \& Carbone 2008; Rimmer, Riley, Wang, Rauworth \& Jurkowsksi, 2004; Roldan \& Reina, 2018). No obstante, en el caso de personas con discapacidad intelectual las barreras tienen que más que ver con factores personales y de percepción de las barreras, como destacan Badía, Orgaz, Verdugo, Ullán \& Martínez (2011), así como la sobreprotección de los adultos; la distancia a los centros donde se realizan las actividades; no tener amigos; o las actitudes de las personas que no tienen discapacidad, incluidos los y las profesionales (Badía \& Longo, 2009). En este último punto, la experiencia previa con personas con discapacidad y el género de los profesionales condicionan las actitudes, de manera que las mujeres tienden a expresar actitudes más positivas hacia la inclusión que los hombres (Hutzler, 2003).

En España, la práctica de AFD para personas con discapacidad intelectual, y en concreto a la Gimnasia Rítmica, se vertebra a través de la Federación Española de Deportes para Personas con Discapacidad Intelectual (FEDDI) y el movimiento Special Olympics Internacional (SOI), con la delegación en España (SOE). Ambas organizan la competición en diversos deportes, entre ellos la gimnasia rítmica, aunque la FEDDI ha desarrollado de manera más específica esta competición. Éstas, junto a las organizaciones deportivas para otros colectivos con discapacidad, organizan los diferentes deportes que acogen atendiendo a la discapacidad de los y las deportistas, no en base a las modalidades deportivas. (Sanz \& Reina, 2012). Son federaciones plurideportivas, y desarrollan sus competiciones al margen de las federaciones de cada uno de los deportes que acogen. En gimnasia rítmica está consolidada la competición gracias a la FEDDI.

En el reglamento de la FEDDI se establecen dos modalidades, la individual, con tres niveles de dificultad, de menor a mayor, denominándose nivel III: Habilidades Deportivas; nivel II: Rítmica Adaptada; nivel I: Rítmica Competición; y la modalidad mixta de conjuntos de 5 gimnastas, con tres niveles de dificultad, de menor a mayor; nivel III: Habilidades Deportivas; nivel II: Rítmica Adaptada; nivel I: Rítmica Competición. En cuanto a edades, existen tres categorías: junior, de 8 a 15 años; absoluto, de 16 a 35 años; y sénior, a partir de 36 años.

En el caso del movimiento SOI, la competición está dividida en los niveles A, B, C, 1, 2 y 3, todos ellos con ejercicios obligatorios que van de menor a mayor complejidad y para los aparatos de pelota, aro, cuerda, mazas y cinta, en función del nivel. Existe un nivel 4, que es el más parecido al reglamento de la FEDDI, ya que se trata de un ejercicio individual y de composición libre, en el que se deben cumplir una serie de requisitos. En la modalidad de conjuntos se desarrollan en grupos de 4 a 6 gimnastas, o de 8 a 12 gimnastas, con ejercicios obligatorios y sin opción a composición libre.

Cabe destacar que fuera de España, la participación de gimnasia rítmica está más difundida bajo el paraguas de SOI. No existe competición a nivel estatal pero se desarrolla en diversos países del mundo movilizando a más 4 millones de deportistas en todos los deportes que organiza. Aunque sobre el impacto que tienen estos eventos no existen apenas evidencias, el estudio liderado por Goodwin, Fitzpatrick, Thurmeier, \& Hall (2006) valorando la perspectiva de las familias de 16 deportistas de 10 a 22 años, concluye que las familias valoraron mucho las explicaciones que les fueron facilitadas; la importancia de mejorar la condición física para incrementar la autonomía; la seguridad que estos eventos les proporcionaron para sus hijos e hijas; y que pudiesen relacionarse con otras personas en entornos controlados. 
Estos aspectos deben tenerse en consideración para tomar decisiones futuras sobre la organización y participación deportiva de deportistas con discapacidad intelectual en inclusión.

Concluyendo, la FEDDI y el movimiento SOI, son organizaciones independientes, con reglamentos específicos y diferentes y no existe en la actualidad relación entre las dos formas de participación.

Por otra parte, cada vez son más las experiencias inclusivas en la práctica de AFD. Ejemplos como el de diferentes entidades públicas como Institut Barcelona Esports, del Ayuntamiento de Barcelona; entidades privadas como la Fundación AVIVA, en Salamanca; la Cátedra Española de Deportes Inclusivos (CEDI), una iniciativa conjunta de la Facultad de Ciencias de la Actividad Física y el DeporteINEF, de la Universidad Politécnica de Madrid y de la Fundación Sanitas, cuyo objetivo principal es fomentar la iniciación y la promoción deportiva en personas con discapacidad a través de contextos inclusivos. Otra iniciativa similar, más local es la que desarrolla la entidad Junts en Acció, en el Garraf, que enfoca sus esfuerzos para que usuarios/as con discapacidad puedan desarrollar programas de actividad física y deportiva en entornos inclusivos, con los apoyos que necesiten.

Se empiezan a dar tímidos pasos en esa dirección por parte del movimiento Special Olympics Catalunya (SOC). Se comienza a concienciar a los clubs deportivos para que creen una Sección Special dentro de la propia estructura del club. Ya hay varios clubs que han puesto en marcha esta sección, con el objeto de incluir deportistas con discapacidad intelectual en sus clubs y compartir el deporte con otros deportistas sin discapacidad. La creación de secciones Special pretende proporcionar un valor añadido a los clubs deportivos, y producir una mejora notable en los deportistas con discapacidad intelectual. (Diari de la discapacitat, 2018)

Existen diferentes modelos de trabajo que facilitan la inclusión y que se pueden ir combinando entre sí. Refiriéndonos a la participación en entrenamientos y competición con deportistas con discapacidad, Sanz \& Reina (2012), proponen un modelo en función de su relación con la actividad física formal y se refieren en primer lugar a Programas segregados, cuando las personas con discapacidad realizan AFD al margen del resto. Por ejemplo la participación en un campeonato de natación organizado para nadadores y nadadoras con discapacidad intelectual; Programas paralelos, cuando se comparten las instalaciones, los materiales, los horarios, pero no la actividad en concreto, con el resto de deportistas; Programas integrados, en los que ambos deportistas, con y sin discapacidad comparten las actividades, con las adaptaciones que sean necesarias para este colectivo; y los Programas inclusivos, los cuales representan una total normalización en la práctica, ya que en ellos no se hace distinción en la intervención en función de las características de las personas que participan.

Existen otros enfoques a partir del planteamiento inclusivo, como el de Van Lent (2006), que distingue cinco niveles de implementación de las AFD desde una perspectiva inclusiva que denomina Espectro Inclusivo, que van desde las actividades separadas o paralelas a las actividades adaptadas, pero siempre se contemplan todas las modalidades dentro del mismo lugar, y tiempo. Esta autora se refiere a Actividades adaptadas, cuando se adaptan materiales, reglas, etc., Actividades paralelas, cuando se realizan paralelamente actividades con adaptaciones; Actividades abiertas, cuando se realizan algunas conjuntamente y otras no; Inclusión inversa, cuando se realizan AFD que son propias de deportistas con discapacidad, como goalball o boccia, con el fin de sensibilizar y conocer estos deportes; y finalmente Actividades separadas, cuando en el mismo club cohabitan ambos colectivos pero en grupos diferentes.

Todas estas modalidades son adecuadas en momentos en los que la situación, las características, la edad, los apoyos necesarios, las competiciones previstas, etc., así lo requieran, ya que no existe un modelo más o menos adecuado a priori. Aunque sí que hay unos principios que hemos intentado respetar, que son los de mantener el máximo tiempo juntos, el máximo tiempo en inclusión, y el máximo de actividades con el mínimo de adaptaciones, como principios imprescindibles para mejorar tanto en el resultado como en las relaciones interpersonales y la actitud. Potenciar el contacto directo es una de las acciones más efectivas para hacer posible que mejore la actitud hacia las personas con discapacidad (Rello, Garoz \& Tejero, 2018),

Finalmente, para el desarrollo de las sesiones, hemos seguido el ejemplo de Vilku \& Tervo, (2006) en Finlandia, y la propuesta de Montilla (2014), en gimnasia artística, en los que se organizan entrenamientos en inclusión, y hemos involucrado a entrenadoras con un perfil educativo y sensibilizadas hacia la propuesta, entendiendo que las habilidades personales de los y las profesionales van a favorecer la inclusión (Canales, Aravena, Cárcamo-Oyarzun, Lorca \& Martínez-Salazar, 2018).

\section{Objetivos de la propuesta}

Dada nuestra realidad, nos proponemos alcanzar los objetivos siguientes en esta experiencia:

- Exponer los ajustes que se han llevado a cabo para disminuir las barreras de participación en los entrenamientos inclusivos de gimnasia rítmica.

Participar en exhibiciones que realiza el club (con coreografías inclusivas y específicas).

- Crear y proponer a la Federación Catalana de Gimnasia, (FCG) un reglamento específico para gimnastas con discapacidad intelectual para incluir un nuevo nivel, el nivel XI, en las competiciones oficiales.

- Participar en competiciones organizadas por la Federación Catalana de Gimnasia, siguiendo el reglamento que se ha propuesto.

\section{Desarrollo de la experiencia}

La experiencia se ha llevado a cabo en un club de Gimnasia Rítmica durante las temporadas 2016-18, comprendidas entre septiembre a julio, a razón de una o dos sesiones semanales de 2 horas cada una, y con el siguiente equipo humano:

Un grupo mixto formado por tres chicas de entre 16 y 21 años, y un chico de 20 años, con discapacidad intelectual y necesidades de apoyo intermitente, siguiendo el paradigma 
de los apoyos. (Verdugo, 1994)

Dos grupos de gimnastas, de categoría infantil y junior, de edades comprendidas entre 12 y 15 años, que entrenan conjuntamente y participan en la modalidad de conjuntos en la Copa Catalana, una competición autonómica. Un total de 15 gimnastas aproximadamente.

Se han desarrollado sesiones en un pabellón deportivo municipal, compartiendo horario de entrenamiento.

Se han implicado dos entrenadoras, que se alternaban entre los días de entrenamiento, una Graduada en Ciencias de la Actividad Física y Deportes y con titulación de entrenadora de nivel autonómico y otra Graduada en Ciencias de la Educación; y proporcionando apoyos esporádicos, una Doctora en Ciencias de la Educación, licenciada en Educación Física, entrenadora de rítmica y especializada en Integración de personas con discapacidad.

\section{Ajustes para disminuir las barreras de participación y aprendizaje}

La cuestión más relevante ha sido determinar los ajustes para disminuir las barreras y favorecer los aprendizajes. Tanto en gimnasia como en otros deportes, Van Lent, Kalvina \& Molik (2006) se refieren a la adaptación de estrategias como herramientas para individualizar las sesiones que deben ser usadas para todos y todas las participantes, no únicamente para los que tienen discapacidad.

\section{Ajustes del reglamento}

Se ha creado un reglamento de gimnasia rítmica denominado Reglamento para gimnastas de ambos sexos con
Tabla 1

Edades y duración de la música para cada categoria. Elaboración propia.

\begin{tabular}{cccc}
\hline & Edad & Duración música & \\
\hline Categorías & & Individual & Conjunto \\
\hline Niños & 8 a 15 anos & 1'00"' a 1'15" & 1'45” a 2'00" \\
\hline Jóvenes & Mayores de 15 años & 1'15'”a 1'30" & 2'15' a 2'30" \\
\hline
\end{tabular}

Tabla 2 .

Elementos de dificultad para los ejercicios individuales. Elaboración propia

Dificultades

Dificultades corporales (BD) 7 dificultades corporales, dos de cada grupo, saltos,

Dificultades corporales (BD) equilibrios, rotaciones y una onda total.

Pasos de danza (S) Mínimo 1 serie de pasos rítmicos de 8” con un elemento técnico de aparato.

Grupos elementos técnicos del Como mínimo 4 grupos de elementos entre los grupos aparato Cónicos fin

Tabla 3.

Elementos de dificultad para los ejercicio de conjunto. Elaboración propia Dificultades Mínimo / Máximo Dificultades corporales (BD) 7 dificultades corporales, dos de cada grupo, saltos, Pasos de danza (S) Mínimo 1 serie de pasos rítmicos de 8”. Como mínimo 4 grupos de elementos entre los grupos Elementos técnicos de aparato técnicos fundamentales y los no fundamentales del aparato \begin{tabular}{ll}
\hline Colaboraciones (cC) & Mínimo 4 colaboraciones.
\end{tabular} Intercambios aparato (ED) Mínimo 1 intercambio donde participen todos los gimnastas.

discapacidad intelectual, Nivel XI presentado a la FCG, que ha sido aprobado por la Junta directiva para su implementación en la temporada 2018. En rasgos generales, su planteamiento correspondería al nivel II del reglamento de la FEDDI: Rítmica adaptada, con 2 pelotas y 3 aros o al nivel 4 del reglamento de SOI.

Se establecen dos posibilidades:

Nivel XI.A. Gimnastas con discapacidad intelectual, en el que es necesario contar con el certificado del grado de discapacidad emitido por el organismo competente. Se puede competir en dos modalidades: modalidad individual masculina o femenina; y modalidad de conjunto: entre 2 y 5

Tabla 4.

Escala descriptiva para evaluar la composición. Elaboración propia

\begin{tabular}{|c|c|c|c|}
\hline Partes composición & $\begin{array}{c}\text { Nivel } 1 \\
\text { 5- } 6 \text { puntos }\end{array}$ & $\begin{array}{c}\text { Nivel } 2 \\
7-8 \text { puntos }\end{array}$ & $\begin{array}{c}\text { Nivel } 3 \\
9-10 \text { puntos }\end{array}$ \\
\hline $\begin{array}{l}\text { Idea Directriz: } \\
\text { CARÁCTER } \\
\text { El estilo y carácter de los movimientos } \\
\text { refleja el carácter de la música; este } \\
\text { carácter conforma una idea guía que se } \\
\text { desarrolla al principio, en el medio y al } \\
\text { final del ejercicio. }\end{array}$ & \begin{tabular}{|l|} 
El carácter de los movimientos no reflejan \\
el carácter de la música. \\
No hay idea guía en gran parte del ejercicio.
\end{tabular} & \begin{tabular}{|l} 
El carácter de los movimientos reflejan el carácter \\
de la música en parte del ejercicio. \\
Hay idea guía desarrollada en parte del ejercicio, \\
no totalmente. \\
El carácter o idea está presente pero no \\
completamente desarrollada en toda la \\
composición.
\end{tabular} & $\begin{array}{l}\text { El estilo y carácter de los movimientos refleja el } \\
\text { carácter de la música. } \\
\text { Hay una idea guía que se desarrolla al principio, en el } \\
\text { medio y al final del ejercicio. } \\
\text { Faltas leves no prolongadas. }\end{array}$ \\
\hline $\begin{array}{l}\text { CONEXIONES } \\
\text { Transiciones lógicas y fluidas de un } \\
\text { movimiento al siguiente }\end{array}$ & $\begin{array}{l}\text { Todo el ejercicio es una serie elementos } \\
\text { desconectados. } \\
\text { No hay transiciones y hay interrupciones } \\
\text { continuas y prolongadas. } \\
\end{array}$ & $\begin{array}{l}\text { Transiciones lógicas y fluidas de un movimiento a } \\
\text { otro en parte del ejercicio. } \\
\text { Algunas interrupciones, pero no muy prolongadas. }\end{array}$ & $\begin{array}{l}\text { Transiciones lógicas. } \\
\text { Transiciones fluidas de un movimiento al siguiente } \\
\text { durante todo el ejercicio . } \\
\text { Faltas leves no prolongadas. }\end{array}$ \\
\hline $\begin{array}{l}\text { RITMO } \\
\text { Armonía entre los movimientos y los } \\
\text { acentos musicales, las frases y el tempo }\end{array}$ & $\begin{array}{l}\text { Todo o gran parte del ejercicio está } \\
\text { desconectado del ritmo (música de fondo) } \\
\text { No sigue tempo, frases, ni acentos. }\end{array}$ & $\begin{array}{l}\text { Alguna falta prolongada en el uso de la música, no } \\
\text { sigue el tempo, frases o acentos. } \\
\text { Pequeñas faltas en varios momentos. }\end{array}$ & $\begin{array}{l}\text { Armonía entre los movimientos y los acentos } \\
\text { musicales, las frases y el tempo durante todo el } \\
\text { ejercicio. } \\
\text { Faltas leves no prolongadas en algún momento de la } \\
\text { música. }\end{array}$ \\
\hline $\begin{array}{l}\text { CAMBIOS DINÁMICOS } \\
\text { La energía, fuerza, velocidad e intensidad } \\
\text { del movimiento se corresponde con los } \\
\text { cambios dinámicos de la música. }\end{array}$ & $\begin{array}{l}\text { Los movimientos de la gimnasta no respetan } \\
\text { los cambios dinámicos o, } \\
\text { La música es totalmente monótona sin } \\
\text { ningún tipo de cambios dinámicos. }\end{array}$ & $\begin{array}{l}\text { La fuerza, la energía, la velocidad y a intensidad } \\
\text { de los movimientos corresponden con los cambios } \\
\text { dinámicos de la música durante parte del ejercicio. } \\
\text { La música es monótona en parte del ejercicio y no } \\
\text { presenta cambios dinámicos. }\end{array}$ & $\begin{array}{l}\text { La fuerza, la energía, la velocidad y a intensidad de } \\
\text { los movimientos corresponden con los cambios } \\
\text { dinámicos de la música durante todo el ejercicio. } \\
\text { La música presenta cambios dinámicos definidos. } \\
\text { Faltas leves no prolongadas. }\end{array}$ \\
\hline EXPRESIÓN CORPORAL & $\begin{array}{l}\text { Movimientos segmentarios, rígidos en todo } \\
\text { el ejercicio. }\end{array}$ & $\begin{array}{l}\text { Participación insuficiente de los segmentos } \\
\text { corporales, rígidos en muchas/ algunas ocasiones. }\end{array}$ & $\begin{array}{l}\text { Movimientos aislados en los que no participan todos } \\
\text { los segmentos corporales. }\end{array}$ \\
\hline $\begin{array}{l}\text { VARIEDAD } \\
\text { Direcciones } \\
\text { Niveles } y \text { desplazamientos } \\
\text { Elementos aparatos }\end{array}$ & $\begin{array}{l}\text { Movimientos ejecutados en la misma zona } \\
\text { del practicable durante mucho tiempo. } \\
\text { Repetición constante de las mismas } \\
\text { direcciones y trayectorias. } \\
\text { No se utiliza todo el practicable. } \\
\text { Nula variedad en el uso de niveles y } \\
\text { modalidades de desplazamiento. } \\
\text { Nula variedad en los planos, direcciones. } \\
\text { No cumple requisitos de elementos de } \\
\text { aparato. } \\
\text { Aparatos mucho tiempo estáticos. } \\
\text { Más de tres elementos decorativos. }\end{array}$ & $\begin{array}{l}\text { Variedad insuficiente en el uso de direcciones y } \\
\text { trayectorias. } \\
\text { Movimientos ejecutados en todo el practicable con } \\
\text { algunas zonas durante más tiempo. } \\
\text { Una zona del practicable no está ocupada. } \\
\text { Variedad insuficiente en el uso de niveles y } \\
\text { modalidades de desplazamiento. } \\
\text { Falta de variedad en los planos, direcciones. } \\
\text { Falta variedad de elementos de aparato Repetición } \\
\text { elementos. }\end{array}$ & $\begin{array}{l}\text { Variedad en el uso de direcciones y trayectorias. } \\
\text { Movimientos ejecutados en todo el practicable. } \\
\text { Todas las zonas del practicable ocupadas. } \\
\text { Variedad en el uso de niveles y modalidades de } \\
\text { desplazamiento. } \\
\text { Variedad en los planos, direcciones. } \\
\text { Variedad de elementos de aparato. } \\
\text { Alguna falta leve. }\end{array}$ \\
\hline $\begin{array}{l}\text { VARIEDAD CONJUNTOS } \\
\text { Formaciones } \\
\text { Intercambios } \\
\text { Colaboraciones } \\
\text { Trabajo colectivo }\end{array}$ & $\begin{array}{l}\text { Menos de } 4 \text { formaciones } \\
\text { Todas formaciones similares. } \\
\text { No se intercambian los aparatos o son muy } \\
\text { sencillos. } \\
\text { Menos de } 4 \text { elementos de colaboración. } \\
\text { Colaboraciones muy similares. } \\
\text { Ausencia de trabajo colectivo (no hay } \\
\text { canon, o coral, o sincronizado) }\end{array}$ & $\begin{array}{l}\text { Falta de variedad en las formaciones, son todas } \\
\text { similares. } \\
\text { Intercambios sencillos pero variados. } \\
\text { Falta de variedad en los elementos de } \\
\text { colaboración. Todos muy similares. } \\
\text { Falta variedad en el trabajo colectivo. }\end{array}$ & $\begin{array}{l}\text { Formaciones diferentes en tipo y amplitud. } \\
\text { Intercambios complejos y variados. } \\
\text { Colaboraciones muy variadas. } \\
\text { Trabajo colectivo variado y complejo. (canon, coral, } \\
\text { sincronizado) en varias ocasiones. }\end{array}$ \\
\hline
\end{tabular}




\begin{tabular}{|c|c|c|c|}
\hline Ejecución & $\begin{array}{c}\text { Nivel } 1 \\
\text { 5-6 puntos }\end{array}$ & $\begin{array}{c}\text { Nivel } 2 \\
7-8 \text { puntos }\end{array}$ & $\begin{array}{c}\text { Nivel } 3 \\
\text { 9-10 puntos }\end{array}$ \\
\hline SALTOS & $\begin{array}{l}\text { Preparación del salto incorrecta. } \\
\text { Impulso insuficiente. } \\
\text { No utiliza brazos en el impulso. } \\
\text { No suficiente altura. } \\
\text { No suficiente amplitud en la forma. } \\
\text { No se aprecia el salto claramente. } \\
\text { Recepción incorrecta: espalda, atrás, bloqueo articulaciones, } \\
\text { brazos descontrolados. } \\
\text { Desequilibrio y caída. }\end{array}$ & $\begin{array}{l}\text { Preparación del salto correcta. } \\
\text { Impulso suficiente. } \\
\text { Brazos no se utilizan correctamente. } \\
\text { Suficiente altura. } \\
\text { Se aprecia parcialmente el salto. } \\
\text { Falta amplitud en la forma. } \\
\text { Recepción parcialmente correcta: pesada, bloqueo de } \\
\text { articulaciones, brazos no participan. } \\
\text { Desequilibrio sin caída. }\end{array}$ & $\begin{array}{l}\text { Preparación del salto con amplitud. } \\
\text { Brazos ayudan al impulso. } \\
\text { Impulso adecuado. } \\
\text { Suficiente altura. } \\
\text { Amplitud en la forma. } \\
\text { Se aprecia claramente la forma del salto. } \\
\text { Utilización de todas articulaciones en todas } \\
\text { las fases. } \\
\text { Recepción adecuada y sin desequilibrios. } \\
\text { Pequeña falta en alguna de las fases del } \\
\text { salto. }\end{array}$ \\
\hline GIROS & $\begin{array}{l}\text { Postura incorrecta de los segmentos corporales. } \\
\text { Preparación incorrecta. } \\
\text { Eje del cuerpo no vertical y desplazamiento sin completar el } \\
\text { giro o caída. } \\
\text { Brazos no participan. } \\
\text { Pierna libre no controlada ni mantenida. } \\
\text { Realización con interrupción y sin amplitud. }\end{array}$ & $\begin{array}{l}\text { Postura parcialmente correcta de los segmentos. } \\
\text { Preparación correcta. } \\
\text { Apoyo del talón durante la realización de giro. } \\
\text { Brazos no participan en todas las fases. } \\
\text { Eje del cuerpo no vertical y termina con paso, colocación } \\
\text { incorrecta. } \\
\text { Pierna libre con amplitud fijada en algún momento. } \\
\text { Saltitos durante el giro. }\end{array}$ & $\begin{array}{l}\text { Postura correcta de los segmentos } \\
\text { corporales y preparación correcta. } \\
\text { Elevación de talón durante el giro. } \\
\text { Participación de brazos. } \\
\text { Eje del cuerpo vertical. } \\
\text { Pierna libre fijada y con amplitud, } \\
\text { colocación adecuada. } \\
\text { Final de giro claramente marcado. } \\
\text { Pequeñas faltas en alguna de las fases del } \\
\text { giro. }\end{array}$ \\
\hline EQUILIBRIOS & $\begin{array}{l}\text { Postura incorrecta de los segmentos corporales. } \\
\text { Forma no fijada ni mantenida. } \\
\text { Apoyo talón en el relevé. } \\
\text { Falta de amplitud, insuficiente en los elementos dinámicos. } \\
\text { Interrupción de los movimientos dinámicos. } \\
\text { Perdida de equilibrio con desplazamiento o caída. }\end{array}$ & \begin{tabular}{|l|} 
Postura parcialmente correcta de los segmentos corporales. \\
Forma fijada pero no mantenida. \\
Apoyo talón/ no elevación suficiente. \\
Amplitud suficiente parcialmente en los elementos \\
dinámicos. \\
Perdida equilibrio con movimiento suplementario con \\
desplazamiento.
\end{tabular} & $\begin{array}{l}\text { Postura correcta de los segmentos } \\
\text { corporales. } \\
\text { Forma fijada y mantenida. } \\
\text { Elevación suficiente y mantenida. } \\
\text { Amplitud máxima en los elementos } \\
\text { dinámicos y continuidad. } \\
\text { Pequeñas faltas en alguna de las fases. }\end{array}$ \\
\hline $\begin{array}{l}\text { TÉCNICA DE BASE } \\
\text { CORPORAL }\end{array}$ & $\begin{array}{l}\text { Postura incorrecta de manera continuada. } \\
\text { Rodillas: descontroladas, con flexión. } \\
\text { Pies y manos: descontrolados. } \\
\text { Brazos/hombros: descolocados y muy flexionados. } \\
\text { Amplitud corporal mínima. } \\
\text { Fuerza y flexibilidad no adecuados. } \\
\text { Descontrol en las acciones técnicas. } \\
\text { Acciones técnicas con muchos errores. } \\
\text { Expresividad nula. }\end{array}$ & $\begin{array}{l}\text { Postura correcta en ocasiones. } \\
\text { Rodillas: alguna vez bien colocadas y extendidas. } \\
\text { Pies y manos: alguna vez controlados. } \\
\text { Brazos/hombros: colocación, amplitud y precisión a veces. } \\
\text { Amplitud corporal a veces. } \\
\text { Fuerza excesiva/insuficiente a veces. } \\
\text { Flexibilidad insuficiente a veces. } \\
\text { Acciones técnicas con algunos errores. } \\
\text { Expresividad en ocasiones. }\end{array}$ & $\begin{array}{l}\text { Alineación corporal y control postural } \\
\text { mantenidos siempre. } \\
\text { Rodillas: controladas, movimientos } \\
\text { precisos.. } \\
\text { Pies y manos: siempre controlados. } \\
\text { Brazos/hombros: colocación, amplitud y } \\
\text { precisión siempre. } \\
\text { Amplitud corporal máxima. } \\
\text { Fuerza y flexibilidad adecuadas a las } \\
\text { exigencias. } \\
\text { Acciones técnicas sin errores. } \\
\text { Expresividad en todas las acciones. } \\
\end{array}$ \\
\hline $\begin{array}{l}\text { ELEMENTOS APARATO } \\
\text { TÉCNICA }\end{array}$ & $\begin{array}{l}\text { Presa incorrecta. } \\
\text { Manejo con crispación } \\
\text { No se percibe el dibujo del aparato, por exceso o falta de } \\
\text { impulso. } \\
\text { No marcan planos, no los mantiene y se cambian con paradas y } \\
\text { alteraciones. } \\
\text { Brusquedad en las acciones, con paradas y sin continuidad. } \\
\text { No hay amplitud, el aparato se sitúa muy cerca del cuerpo. } \\
\text { Muchos momentos el aparato está parado, estático. } \\
\text { Aparato cae a menudo. } \\
\text { Expresión desigual y/o inapropiada. }\end{array}$ & $\begin{array}{l}\text { Presa correcta con errores de crispación o imprecisión. } \\
\text { Toca al suelo o cuerpo ligeramente. } \\
\text { Marca los planos y el dibujo con imprecisiones (exceso o } \\
\text { falta de impulso). } \\
\text { Mantiene los planos pero los cambia con imprecisiones, } \\
\text { alteraciones del dibujo o bruscamente. } \\
\text { Acciones con continuad, alguna vez hay paradas. } \\
\text { Las acciones se realizan con poca de amplitud y poca } \\
\text { participación del aparato. } \\
\text { Aparato cae alguna vez. } \\
\text { Expresión desigual i/o inapropiada en ocasiones. }\end{array}$ & $\begin{array}{l}\text { Presa correcta y sin crispación. } \\
\text { Dibujo claramente definido. } \\
\text { Aparato no toca suelo ni cuerpo. } \\
\text { Marca planos claramente. } \\
\text { Cambios de plano con continuidad, claridad } \\
\text { y precisión. } \\
\text { Continuidad siempre, no permanece } \\
\text { parado. } \\
\text { El aparato se sitúa alejado, se implica a } \\
\text { todo el cuerpo en las acciones, hay } \\
\text { amplitud. } \\
\text { El aparato siempre participa en las } \\
\text { acciones. } \\
\text { Expresión igual y excelente en todos los } \\
\text { participantes. }\end{array}$ \\
\hline TRABAJO COLECTIVO & $\begin{array}{l}\text { Desincronizados durante toda el ejercicio (modalidad parejas } \\
\text {-grupos): } \\
\text { en música, en formaciones, en acciones corporales y con los } \\
\text { aparatos, en los desplazamientos, en los intercambios, en los } \\
\text { elementos de colaboración y trabajo colectivo. }\end{array}$ & $\begin{array}{l}\text { En diversas ocasiones hay errores de sincronización } \\
\text { (modalidad parejas -grupos): } \\
\text { en música, en formaciones, en acciones corporales y con } \\
\text { los aparatos, en los desplazamientos, en los intercambios, } \\
\text { en los elementos de colaboración y trabajo colectivo. }\end{array}$ & $\begin{array}{l}\text { Sincronizados } \\
\text { (modalidad parejas -grupos): } \\
\text { en música, en formaciones, en acciones } \\
\text { corporales y con los aparatos, en los } \\
\text { desplazamientos, en los intercambios, en } \\
\text { los elementos de colaboración y trabajo } \\
\text { colectivo. } \\
\text { Pequeñas faltes en algunas ocasiones. }\end{array}$ \\
\hline
\end{tabular}

gimnastas, todos del mismo género o mixtos.

Nivel XI.B. Conjuntos unificados, con la incorporación de gimnastas con y sin discapacidad en el mismo conjunto. A partir de 2 a 6 gimnastas, siendo el número de gimnastas sin discapacidad la mitad o menos que con discapacidad.

Se establecen dos categorías por edad y la duración de las músicas se reduce. (Tabla 1). Respecto a los aparatos, para la modalidad individual, serán libres, adecuando la elección a cada gimnasta. En conjuntos se utilizan pelotas y aros, rígidos y con muchas opciones de manipulación en este nivel.

Se han reducido en cantidad y dificultad de los elementos corporales respecto al reglamento oficial de gimnasia rítmica y se han eliminado las penalizaciones. (Tablas 2 y 3). Se han elaborado dos escalas descriptivas (Tablas 4 y 5 ) en las que se establecen tres posibilidades de puntuación, que van desde 5 a 10 puntos. Un ejercicio tendrá una puntuación de 5 puntos como mínimo. Esta escala será utilizada por los y las jueces y puede convertirse en una importante herramienta. Con ello pretendemos valorar el esfuerzo realizado en los entrenamientos, además de la presentación el día de la competición. Creemos que los y las gimnastas deben percibir que su trabajo es valorado con una puntuación que mentalmente se acerque a lo correcto, para incidir en la motivación y la continuidad de la actividad. Para profundizar más en el reglamento presentado instamos a consultarlo en la web de laFCG.

\section{Ajustes del espacio de entrenamiento y material}

No hemos modificado el tapiz de 13x13, que hemos usado en los entrenamientos y en las exhibiciones como el resto de gimnastas, sí que hemos incidido en la ocupación del mismo. Debido a la menor capacidad para desplazarse, la variedad y la riqueza en la ocupación del espacio se ha visto modificada, las trayectorias han sido más cortas y lentas, las formaciones se han mantenido más tiempo y se ha optado por formas sencillas y geométricas como rectas, cuadrados, en las que se pueden orientar más fácilmente.

En segundo lugar, hemos utilizado aros y pelotas, por ser aparatos muy versátiles que propician habilidades como sal- 
tar, rotar, pasar por dentro, rodar, etc. (Cassagne, 1990) y con gran atracción por parte de los y las gimnastas. Hemos disminuido el tamaño utilizando aros de $70 \mathrm{~cm}$ de diámetro, que facilita la manipulación y dominio.

En cuanto a la música, ha sido seleccionada teniendo en cuenta el tempo, unos 100 pulsaciones por minuto (ppm), con una estructura sencilla de $4 / 4$, y con pulsaciones muy marcadas. El uso de las palmadas para marcar el tempo en la realización de los elementos, acentuado con el uso de la voz para contar los números de los tiempos musicales ha ayudado a la comprensión y ejecución de las acciones.

\section{Ajustes en la organización de las sesiones}

Hemos tenido en cuenta diferentes tipos de organización en las sesiones y en la participación de eventos. De acuerdo con Sanz \& Reina (2012), y Van Lent (2006), por un lado hemos realizado entrenamientos en inclusión o abiertos, como los calentamientos, la parte principal de técnica de danza, o desarrollo de elementos grupales como elementos de colaboración, o formaciones, para la composición de la coreografía de los festivales inclusivos del club. En otros momentos, se han desarrollado partes del entrenamiento conjuntamente pero con tareas adaptadas, y en otros, se han realizado actividades separadas, en los que se ha creado un ejercicio específico, o se han entrenado elementos de las competiciones. Por tanto, se han organizado los entrenamientos de diversas formas, pasando de la inclusión, adaptando las acciones a las capacidad de los y las gimnastas con discapacidad; a las actividades separadas, para la creación de coreografías siguiendo una normativa específica. En todos los casos, la presencia de las dos entrenadoras ha jugado un rol importante. Una en cuanto a la impartición de las sesiones y la otra en cuanto al refuerzo, apoyo y ajustes individuales.

\section{Consideraciones sobre la metodología}

Por un lado la gimnasia se basa en la reproducción de formas definidas con un modelo determinado por el reglamento, y por otro, en la producción de enlaces que configuran coreografías únicas y originales.

La metodología contempla el estilo de enseñanza y el estilo de comunicación, que es otro de los aspectos que Van Lent, Kalvina \& Molik, (2006) mencionan como fundamentales para el éxito de las actividades. Con esta realidad, en las sesiones de entrenamiento, es necesario alternar metodologías basadas en la reproducción y en la producción, con algunas modificaciones (Kaufman, 2006; GuerberWalsh, Leray \& Maucouvert, 2000), para ir consiguiendo poco a poco avances en ambos sentidos. Teniendo en cuenta que en gimnasia rítmica recibimos información por diferentes vías (visual, auditiva, kinestésica y táctil), hemos ido modificando las instrucciones en función de los objetivos y de las características de nuestros y nuestras gimnastas, por lo que hemos introduciendo de manera jerarquizada los siguientes tipos de instrucciones (Kaufman, 2006; Van Lent, Kalvina \& Molik, 2006; Guerber- Walsh, Leray \& Maucouvert, (2000):

1. Instrucciones verbales, incidiendo en la percepción auditiva. Hemos realizado descripciones de lo que pretendemos que los y las gimnastas realicen. Puede ser referido a un elemento en concreto (reproducción de un equilibrio en passé) o a la propuesta de un elemento libre o un enlace (producción de un lanzamiento libre). Se han realizado instrucciones cortas, y claras, con lenguaje sencillo e introduciendo el vocabulario específico de la gimnasia rítmica.

2. Demostraciones, incidiendo en la percepción visual. Esta estrategia se ha utilizado cuando no se ha entendido con instrucción verbal. Es muy importante que los y las gimnastas comprenden lo que ven y lo que escuchan. Si las demostraciones las acompañamos con instrucciones verbales reforzamos el mensaje. Cuando pretendemos que se reproduzca un modelo preciso, como por ejemplo realizar un equilibrio con el aro rotando alrededor del cuello, esta ha sido una de las estrategias más utilizadas. Y el rol de la entrenadora de apoyo junto con otras gimnastas ha sido muy importante.

3. Instrucciones kinestésicas y con contacto físico, incidiendo en la percepción kinestésica. Se ha recurrido a esta estrategia cuando no ha sido capaz de realizar la tarea que se le ha solicitado con las anteriores indicaciones. Son indicaciones con el contacto físico sobre la posición de las diferentes partes del cuerpo para ir adecuándose al modelo que pretendemos ejecutar, que pueden ir acompañadas de instrucciones verbales.

Estos niveles de instrucción van de menos a más intrusivos y hemos ido utilizando un tipo u otro de instrucción cuando el anterior ha fracasado, ya que no perdemos de vista que los y las gimnastas han de ir mejorando su autonomía y control corporal y llegar a realizar los ejercicios solos. La observación directa de las dos entrenadoras es el método que se ha utilizado para determinar qué tipo de información se les proporcionaba, y a quién, ya que nos todos los y las gimnastas presentaban las mismas capacidades para el aprendizaje.

Para este planteamiento han participado además gimnastas sin discapacidad, creando así un clima de trabajo cooperativo, algo que nos interesaba mucho y que ha favorecido las relaciones interpersonales, como también exponen Kaufman, (2006) y Van Lent, Kalvina \& Molik, (2006), denominándolo interacciones en parejas. Otra acción ha sido implicarles en la toma de decisiones, como decidir si querían participar juntos o separados en los festivales, las fechas, el vestuario, etc., promoviendo de este modo la inclusión en todos los momentos.

Es muy interesante destacar que en gimnasia rítmica, cuando se desarrollan proyectos coreográficos en grupo, todas las relaciones que se establecen entre los componentes del conjunto son de colaboración, ya se que se trabaja para un fin común, un ejercicio único y efímero que será mostrado al público. El hecho de crear coreografías con gimnastas con diferentes niveles de comprensión y ejecución aporta un valor añadido, la aceptación de diferentes niveles y capacidades y la organización de diversos roles dentro de la coreografía conjunta. Estas tareas las ha desarrollado perfectamente la entrenadora implicada, la cual, desde el principio de este proyecto ha tenido un posicionamiento y determinación claros para garantizar la participación de todos y todas, así como la implicación en la toma de decisiones, contribuyendo claramente de las dimensiones relacionadas con autodeterminación, relaciones interpersonales e 
inclusión social (Shalock \& Verdugo, 2003).

Siguiendo con la metodología, otra de las acciones que hemos realizado es la confección de dibujos, fotos, y videos, para darle significación a la coreografía y a la composición que hemos ido elaborando. Se ha recurrido a la elaboración de esquemas coreográficos, con dibujos y colores para cada parte del tema musical, así como la descripción gráfica de las partes de la coreografía con las formaciones, roles para cada gimnasta y transiciones, elaborados a mano, con la intención de mejorar la compresión. Se han usado en algunos momentos de las sesiones, y han servido de guía para comprender y memorizar la coreografía. Se realizaba una lectura del esquema, por parte de los y las gimnastas, describiendo qué elementos y acciones correspondían a cada sección (Figura 1$)$.

Una vez dadas las instrucciones, hemos valorado las respuestas posibles así como las conductas que podían aparecen en las sesiones, para tener preparadas las estrategias o respuestas a las conductas menos adecuadas de los y las gimnastas. Hemos tomado en consideración y como referencia el Apoyo Conductual Positivo de Goñi, Martinez \& Zardoya (2007), de manera que hemos intentado reforzar positivamente las respuestas adecuadas y ajustadas a los requisitos. Destacamos la escasez de respuestas desajustadas o problemáticas, quizá debido a que el grupo se conoce desde hace muchos años, o porque asisten a las sesiones por motivación propia.

Después de algunas sesiones de entrenamiento, para facilitar la compresión y ejecución de las coreografías, una de las entrenadoras ha pasado a formar parte del conjunto, creando de esta manera un apoyo específico dentro del grupo. Hemos convertido el conjunto en mixto unificado. Este hecho nos ha ayudado a relajar a los y las gimnastas, ya que comprobamos que su capacidad para memorizar y ejecutar todo el conjunto de elementos, enlaces, seguir la música, tener en cuenta al compañero, etc., era limitada y se acumulaban demasiados estímulos para controlar, generaba a veces tensión, entre otras razones, por la cercanía de las presentaciones y exhibiciones.

Una vez finalizado el trabajo creativo de la composición del ejercicio, se han realizado una serie de acciones para que fuera más fácilmente recordado. En primer lugar se han ido filmando por partes y se les ha enviado al grupo utilizando wasap, con la finalidad de que pudiesen visualizarse en casa. Por otro lado, se ha dividido la coreografía en partes y ha entrenado cada parte de forma separada para dedicar el mis-

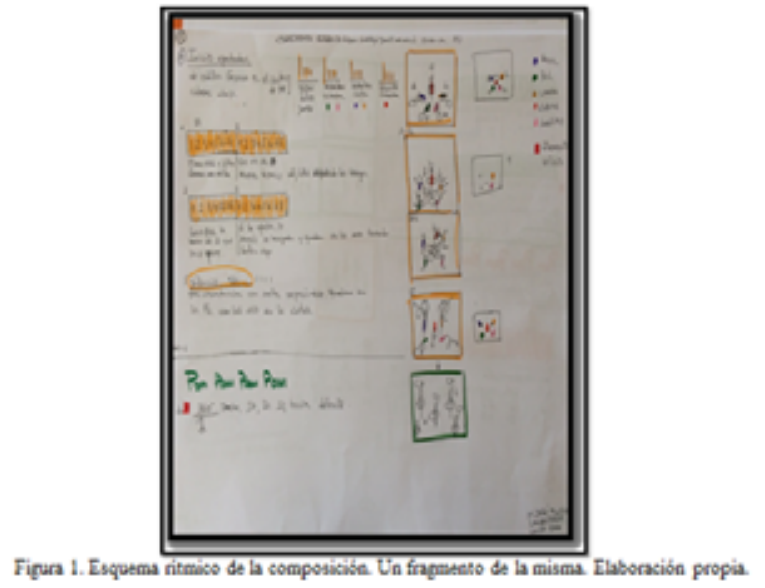

mo tiempo a cada una de ellas. En definitiva, que con todas las adaptaciones y estrategias expuestas se ha podido garantizar la participación en todas las sesiones y eventos, como exponemos a continuación.

\section{Resultados de la experiencia y discusión}

El primer y segundo objetivo, exponer los ajustes que se han llevado a cabo para disminuir las barreras de participación en los entrenamientos inclusivos de gimnasia rítmica para formar parte de los entrenamientos en gimnasia rítmica en inclusión con la implicación del resto de gimnastas, los hemos alcanzado de forma muy satisfactoria. Hemos de decir que la experiencia ha resultado más sencilla de implementar de lo que habíamos previsto, y con una gran implicación por parte de todos los y las gimnastas. Parece que la experiencia de ponerse en el lugar de gimnastas con discapacidad y convivir con ellos, provoca un cambio en la actitud hacia este colectivo, como exponen Rello, Garoz \& Tejero (2018), acentuado por el hecho de haberse producido de manera continuada durante dos temporadas.

Una de las razones puede ser el esfuerzo de las entrenadoras para implicar a todas las gimnastas para empatizar con los y las gimnastas con discapacidad. Hemos comprobado que dar responsabilidad, que hacerles sentir parte de un grupo junto con otras gimnastas les ha hecho crecer el sentimiento de pertenencia y la motivación necesarios para abordar un trabajo de este tipo. Hemos comprobado cómo las gimnastas sin discapacidad han aceptado e incorporado fácilmente a la rutina de los entrenamientos a todos los nuevos compañeros y compañeras y se han convertido en verdaderas redes de apoyo, corroborando los estudios de Abellán, Sáez-Gallego \& Reina (2018).

El segundo de los objetivos, participar en exhibiciones que realiza el club -con coreografías inclusivas y específicas-, también ha sido conseguido. Fruto de todo el trabajo realizado, el grupo ha participado en una coreografía conjunta con las gimnastas sin discapacidad, en la exhibición de Navidad del Club. Con un ejercicio específico con aros, en la modalidad mixta unificada, ha participado como exhibición en un Trofeo del Club; y en dos exhibiciones durante competiciones oficiales de la FCG, junto con dos grupos más de gimnastas con discapacidad de otro club.

En todos estos eventos hemos explicado al público asistente la importancia que para el colectivo con discapacidad tiene la inclusión deportiva. La difusión ha sido grande ya que en todos la asistencia de público ha sido muy elevada. Es muy probable que, aunque no podemos asegurarlo, la presencia de gimnastas con discapacidad propicie la actitud positiva hacia las personas con discapacidad, como señalan Rello, Garoz \& Tejero, 2018) y mejore participación normalizada en el deporte de todo tipo de deportistas. Incluir a este colectivo en la vida deportiva es seguir un modelo inclusivo de sociedad.

El objetivo de crear un reglamento específico para gimnastas con discapacidad intelectual para incluir un nuevo nivel en las competiciones oficiales también lo hemos conseguido. La FCG ha aceptado la propuesta del nuevo reglamento, para que gimnastas con discapacidad participen a partir de 2018 en la competición oficial. Se denomina 
Nivel XI, como uno más dentro de sus niveles de competición, que hasta ahora acababa en el nivel X. Probablemente, se amplíe a otras disciplinas como la gimnasia artística, acrobática, o aeróbic. Otro paso importante hacia la unificación y la inclusión en el deporte.

El último de los objetivos era participar en competiciones organizadas por la Federación Catalana de Gimnasia, siguiendo el reglamento que se ha creado. Esta primera competición se ha celebrado el día 21 de Octubre, bajo el nombre de I Copa Catalana de Gimnasia Rítmica para gimnastas con discapacidad intelectual, ( Nivel XI ) con la participación de cuatro conjuntos, uno mixto y tres formados por gimnastas de género femenino. Con un total de 20 gimnastas, en el marco de la $2^{\mathrm{a}}$ Fase de Copa Catalana de conjuntos y junto a más de 1000 gimnastas sin discapacidad, de 60 Clubs de Cataluña. Esperemos que sólo sea el inicio.

Esperemos también que en un futuro no muy lejano sean todas las federaciones deportivas las responsables del entrenamiento y competición de todas y todos los deportistas, incluidos los que tienen alguna discapacidad. Ya que son éstas las que tienen la responsabilidad en la organización y promoción de los deportes. En nuestra opinión, debería ser una obligación para con todos y todas, tal y como marca la Convención de la ONU de 2006.

Este paso lo han dado ya algunas federaciones, como es el caso de la Federación Española de Taekwondo, en la incorporación y la regulación de la modalidad Parataekwondo; la de Tenis Mesa, Piragüismo, o Judo; que tienen secciones específicas para deportistas, sobre todo con discapacidades sensoriales o físicas.

Aunque el impacto sobre las y los participantes es un tema que se deberá seguir estudiando, sin duda los argumentos que nos proporcionan los diversos estudios presentados han de servir de guía para garantizar la participación. Lo que parece evidente es que juntos es mejor que separados, siguiendo el paradigma inclusivo.

\section{Reflexiones finales y perspectivas de futuro}

No cabe duda que durante estos años de trabajo han ido apareciendo nuevas inquietudes y retos para abordar. En primer lugar, la Federación Catalana de Gimnasia ha aceptado la propuesta de nuevo reglamento que nos ha permitido participar, a partir del 2018 a gimnastas con discapacidad, en las competiciones autonómicas. Realizar un seguimiento de la aceptación e impacto de este nuevo reglamento en los y las gimnastas con discapacidad es un tema que creemos de gran relevancia, es pionera en este deporte. La propuesta tiene una repercusión en toda Cataluña, y esperemos que en otras comunidades,

Por otra parte, contar con un reglamento específico, nos permite programar los entrenamientos de manera más coherente para los y las gimnastas con discapacidad intelectual. Hemos evidenciado que es necesario aumentar los días de entrenamiento, ya que el tiempo útil de un entrenamiento semanal no es suficiente para consolidar los aprendizajes. Aumentamos para la temporada 2019 a dos días por semana y algunoa sábados.

Otras acciones que creemos necesarias es llevar a cabo una campaña de difusión para aumentar el número de gimnastas con discapacidad en el club, actualmente solo cuatro de más de doscientas gimnastas. Para demostrar que es positivo y necesario dar este paso hacia la inclusión, en un futuro no lejano deberíamos analizar el impacto que el trabajo en inclusión tiene sobre los agentes que intervienen, entrenadoras, jueces, familias y gimnastas sin discapacidad, familias y gimnastas con discapacidad.

Por otra parte, difundir este modelo de trabajo en entornos normalizados a otras entidades e implementar la modalidad deportiva de gimnasia rítmica para gimnastas con discapacidad intelectual. Se abre una línea clara de investigación que puede ser pionera en este campo Montilla \& Pérez, (2017). Analizar el impacto, los beneficios, los obstáculos y los ajustes necesarios desde el punto de vista metodológico, para demostrar que la inclusión en deporte también es positiva, al igual que en otros ámbitos de la vida, como la educación o el trabajo, como ya se está evidenciando. Así como proporcionar herramientas a otras personas que puedan estar interesadas en implementar este modelo, con una propuesta metodológica para el desarrollo de los entrenamientos en inclusión.

Finalmente, estamos convencidos de que estamos contribuyendo a mejorar la calidad de vida de las gimnastas con discapacidad y otro aspecto muy importante, la actitud del resto de la sociedad hacia este colectivo. A raíz de la inclusión en la vida deportiva del club de los y las gimnastas con discapacidad, hemos recibido muchas muestras de entusiasmo y de admiración, por parte de otros entrenadores y familias. Es esperanzador, y esperamos de esta manera poder ayudar a que la inclusión deportiva, poco a poco, sea una realidad, no únicamente en la gimnasia rítmica, sino en todos los deportes.

\section{Agradecimientos}

Este trabajo ha podido llevarse a cabo gracias a la participación de los y las gimnastas Aina, Gemma, Marina y Pol. Son fantásticos, siempre dispuestos a participar en todas las sesiones y actuaciones que les hemos propuesto. A Laura Pérez, por creer en el proyecto, por dar los pasos y tocar las teclas adecuadas. Sin su valiosa participación no habría sido posible. A Laura Porras, por su cariño e implicación como entrenadora y como gimnasta. No ha dudado ni un momento en participar como gimnasta para facilitar los aprendizajes y su ejecución final. A las gimnastas que nos han arropado, comprendido y participado en todo el proceso. El futuro está garantizado con su calidad humana. Y finalmente, gracias a las familias de los y las gimnastas, por no dudar, por dudar, por colaborar, por escuchar, por acompañarnos en todo el proceso. Gracias.

Con el apoyo del Instituto Nacional de Educación Física de Catalunya (INEFC) de la Generalitat de Catalunya.

\section{Referencias}

Abellán, J., Sáez-Gallego, N. \& Reina, R. (2018). Explorando el efecto del contacto y el deporte inclusivo en Educación Física en las actitudes hacia la discapacidad intelectual en estudiantes de secundaria. RICYDE. Revista Internacional de Ciencias del Deporte, 14 (34), 233-242. doi:10.5232/ricyde

Badía, M. \& Longo, E. (2009). El ocio en las personas con discapacidad 
intelectual: participación y calidad de vida a través de las actividades de ocio. Siglo Cero, 40 (3), 30-44.

Badía, M., Orgaz, B. M., Verdugo, M. A., Ullán, A. M. \& Martínez M.M. (2011). Personal factors and perceived barriers to participation in leisure activities for young and adults with developmental disabilities. Research in developmental disabilities, 32 (6), 2055-63. doi: 10.1016/j.ridd.2011.08.007.

Canales, P., Aravena, O., Carcamo-Oyarzun, J., Lorca, J. \& MartinezSalazar, C. (2018). Prácticas pedagógicas que favorecen u obstaculizan la inclusión educativa en el aula de educación física desde la perspectiva del alumnado y profesorado. Retos. Nuevas tendencias en Educación Física, Deporte y Recreación, 34, 212217. Recuperado de: https://recyt.fecyt.es/index.php/retos/article/ view/59620.

Cassagne, Paris. Anphora.

Cátedra Estudios de Deporte Inclusivo. CEDI. (2018). Recuperado de: https://www.deporteinclusivo.com.

Diari de la discapacitat. (2018). Més de 400 persones participen al Simpòsium sobre la inclusió en els clubs esportius. Recuperado de: http://diarideladiscapacitat.cat/mes-de-400-persones-participenal-simposium-sobre-la-inclusio-en-els-clubs-esportius/.

Espósito, P.E., MacDonald, M., Hornyak, J.E. \& Urich, D.A. (2012). Physical activity patterns of youth with Down síndrome. Intellectual and developmental disabilities, 50(2),109-119. doi: 10.1352/1934-9556-50.2.109.

Federation International de Gymnastique. (2017). Código de Puntuación de Gimnasia Rítmica. Recuperado de: https://fedegim.info/ docWeb/19/2012/2016/GR/RFEG/RG_CoP_2017-2020_sp.pdf.

Goñi, Mª. J., Maño, N. \& Zardoya, A. (2007). Apoyo conductual positivo. Algunas herramientas para afrontar conductas difíciles. Cuadernos de buenas prácticas. Madrid. FEAPS.

Goodwin, D., Fitzpatrick, D., Thurmeier, R. \& Hall, C. (2006). The decisión to join Special olympic. Parent's perspective. Adapted Physical Activities Quarterly, 23(2), 163-183. doi: http:// dx.doi.org /10.1123/apaq.23.2.163.

Guerber- Walsh, N., Leray, C. \& Maucouvert, A. (2000). Danza. De la escuela a las asociaciones. Lleida. Agonos.

Hutzler, Y. (2003). Attitude toward the participation of individuals with disabilities in physical activity: A reviews. QUEST, SS, 347-373. https://doi.org/10.1080/00336297.2003.10491809

Hutzler, Y. \& Korsensky, O. (2010). Motivational correlates of physical activity in persons with an intellectual disability: a systematic literature review. Journal of Intellectual Disability Research, 54 (9), 767-786. doi: https://doi.org/10.1080/ 00336297.2003.10491809

Izquierdo-Goìmez, R. \& Díaz-Cueto, M. (2017). Joven con síndrome de Down y natación recreativa: posibilidades hacia la inclusión. Revista Internacional de Medicina y Ciencias de la Actividad Fiìsica y el Deporte, 17(65), 43-62. Recuperado de: https:// revistas.uam.es/rimcafd/article/view/7357. doi: http://dx.doi.org/ 10.15366/rimcafd2017.65.003.

Kaufman, K. (2006). Inclusive Creative movement and dance. USA. Human Kinetics.

Roldan, A. \& Reina, R. ( 2018). Deporte escolar y universitario en las personas con discapacidad. En Libro Blanco del deporte con personas con discapacidad en España. (pp 181-209). Madrid. Cinca. [Versión X Reader]. Recuperdado de: http://sid.usal.es/idocs/ F8/FDO27470/Libro_blanco_deporte.pdf

Montilla, Ma ${ }^{a}$ J. (2014). Deporte e inclusión. Una experiencia en gimnasia artística con gimnastas con discapacidad intelectual. En Pérez, J. \& Reina, R. European Congress of Adapted Physical Activity. Libro de Actas del congreso. Serie «Publicaciones del CEDI». Madrid.

Montilla, Ma J. \& Pérez, L. (2017). Deporte e inclusión. El caso de un grupo de gimnastas con discapacidad intelectual en gimnasia rítmica. En Rodríguez, E., Alcaraz. V., Ruiz, F., Prada \& Pérez, A.(Ed) I Congreso Internacional de Discapacidad Intelectual, Actividad Física y Salud. Sevilla: Universidad Pablo de Olavide / Universidad de Sevilla (pp 158 -163 ). Sevilla.

Reglamento Nivel XI. Gimnastica rítmica.

Gimnastes amb discapacitats intel-lectuals o del desenvolupament. Recuperado de: https://fedegim.info/docWeb/19/2012/2018/GR/ COMPETICIONS_NIVELL_XI_GIMNASTES_AMB_DISCAPACITAT_.pdf Elementos corporales Nivel XI. Gimnastica rítmica.
Gimnastes amb discapacitats intel-lectuals o del desenvolupament. Recuperado de: https:/fedegim.info/docWeb/19/2012/2018/GR/ ELEMENIS_CORPORALS_NIVEL_XI_GIMANASIES_AMB_DISCAPACITATIPdf.

Moya-Mata, I., Ruiz, L., Martin, J., Pérez, P. Mª \& Ros, C. (2017). La representación de la discapacidad en las imágenes de los libros de texto de Educación Física: ¿inclusión o exclusión?. Retos. Nuevas tendencias en Educación Física, Deporte y Recreación, 32, 88-95. Recuperado de: https://www.redalyc.org/pdf/3457/ 345751100018.pdf

Murphy, N. A., \& Carbone, P. S. (2008). Promoting the participation of children with disabilities in sports, recreation, and physical activities. Pediatrics, 121(5), 1057-1061. doi: 10.1542/ peds.2008-0566.

Organización de Naciones Unidas. (2006). Convención sobre derechos de las personas con discapacidad. Recuperado de: http:// www.un.org/spanish/disabilities/default.asp?id=497

Pimenta, R.A., Zuchetto, A.T., Bastos, T. \& Corredoira, R. (2016). Efectos de la natacioìn para joìvenes con trastorno del espectro autista. Revista Internacional de Medicina y Ciencias de la Actividad Física y el Deporte, 16 (64), 789-806. Recuperado de: https://repositorio.uam.es/handle/10486/676197. doi: http:// dx.doi.org/10.15366/rimcafd2016.64.011.

Ramos, P., Jimeìnez-Iglesias, A., Rivera, F. \& Moreno, C. (2016). Evolución de la práctica de la actividad física en los adolescentes españoles. Revista Internacional de Medicina y Ciencias de la Actividad Física y el Deporte. vol. 16(62), 335-353. doi: http:/ /dx.doi.org/10.15366/rimcafd2016.62.010.

Rello, C., Garoz, I. \& Tejero, C. Ma . (2018). Análisis comparativo del efecto de tres programas de sensibilización hacia la discapacidad en Educación Física. Retos. Nuevas tendencias en Educación Física, Deporte y Recreación, 3, 88-95. Recuperado de: https:// recyt.fecyt.es/index.php/retos/article/view/59889

Rimmer, J. H., Riley, B., Wang, E., Rauworth, A. \& Jurkowsksi, J. (2004). Physical activity participation among persons with disabilities: barriers and facilitators. American Journal of Preventative Medicine, 26, 419-425.

Rodriìguez, J., Castillo, D., Redin S. \& Yanci, J. (2016). Análisis de la actividad física extraescolar realizada por adolescentes con discapacidad intelectual. En Gallego, J., Alcaraz, M., Aguilar J.M., Cangas, A.J. \& Martínez, D. (Ed.) Avances en actividad física y deportiva inclusiva. (pp. 130-138). Almería. Coleccion: Libros electronicos $n^{\circ} 58$.

Sanz, D. \& Reina, R. (2012). Actividades físicas y deportes adaptados para personas con discapacidad. Barcelona. Paidotribo.

Shalock, R. \& Verdugo, M.A. (2003). Calidad de vida. Manual para profesionales de la educación, salud y servicios sociales. Madrid. Alianza editorial.

Special Olympics España (n.d.) Recuperado de: http:// w w w. s p e c i a l o l y m p i c s. e s/ index.php?option=com_contentyview $=$ featuredyItemid=101.

Reglamento de gimnasia Rítmica adaptada (2018). Federación Española de deportes para personas con discapacidad intelectual. Recuperado de: http://www.feddi.org/ DeportesCirculares.asp?id=7ydeporte=Gimnasia\%20Ritmica.

Rhythmic Gymnastics Routines (2018). Recuperado de:https:// resources.specialolympics.org/Taxonomy/Sports_Essentials/ Rhythmic_Gymnastics/Rhythmic_Gymnastics_Routines_(20162023)-OLD.aspx

Van Lent, Kalvina \& Molik (2006). Adaptation strategies. En Van Copennolle, H. (Coor.). Count me in. A guide to inclusive physical activity, sport and leisure for children with a disability. (pp.33$60)$. Belgium. Faculty of kinesiology and rehabilitation Sciences.

Verdugo, M.A. (1994). El cambio de paradigma en la concepción del retraso mental: la nueva definición de la AAMR. Siglo Cero, 25 (5), 5-24.

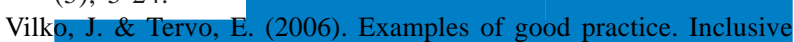
, J. \& Tervo, E. (2006). Examples of good practice. Inclusive gymnastics. En Van Copennolle, H. (Coor.). Count me in. A
guide to inclusive physical activity, sport and leisure for children
with a disability. (pp.74-77). Belgium. Faculty 